\title{
Absence of Spontaneous Magnetic Order at Nonzero Temperature in One- and Two-Dimensional Heisenberg and $X Y$ Systems with Long-Range Interactions
}

\author{
P. Bruno* \\ Max-Planck-Institut für Mikrostrukturphysik, Weinberg 2, D-06120 Halle, Germany
}

(Received 6 May 2001; published 7 September 2001)

\begin{abstract}
The Mermin-Wagner theorem is strengthened so as to rule out magnetic long-range order at $T>0$ in one- or two-dimensional Heisenberg and $X Y$ systems with long-range interactions decreasing as $R^{-\alpha}$ with a sufficiently large exponent $\alpha$. For oscillatory interactions, ferromagnetic long-range order at $T>0$ is ruled out if $\alpha \geq 1(D=1)$ or $\alpha>5 / 2(D=2)$. For systems with monotonically decreasing interactions, ferro- or antiferromagnetic long-range order at $T>0$ is ruled out if $\alpha \geq 2 D$.
\end{abstract}

In a seminal paper exploiting a thermodynamic inequality due to Bogoliubov [1], Mermin and Wagner proved the following important theorem [2]: "For one- or twodimensional Heisenberg systems with isotropic interactions, and such that the interactions are short ranged, namely, which satisfy the condition

$$
\sum_{\mathbf{R}} \mathbf{R}^{2}\left|J_{\mathbf{R}}\right|<+\infty
$$

there can be no spontaneous ferro- or antiferromagnetic long-range order at $T>0$ [3]." In view of the fact that most of our knowledge of critical phenomena and phase transitions is based upon approximate theories, the few known rigorous results [4] such as the Mermin-Wagner theorem are of key importance, for they allow one to test the validity of approximate theories.

For interactions with a finite range or with an exponential decay, the condition (1) is trivially satisfied. For interactions with a power-law decay $\left|J_{\mathbf{R}}\right| \propto R^{-\alpha}$, condition (1) is satisfied provided that $\alpha>D+2$, where $D$ is the dimensionality. In metallic magnetic systems, the exchange interactions are of the Ruderman-Kittel-KasuyaYosida (RKKY) type [5], which have a long-range oscillatory behavior for large $R: \quad J_{\mathbf{R}} \propto R^{-D} \cos \left(q_{0} R+\phi\right)$. It is obvious that the RKKY interactions do not satisfy the criterion of short rangedness (1), so that no conclusion on the magnetism one- and two-dimensional RKKY systems can be obtained from the Mermin-Wagner theorem. This situation is highly unsatisfactory, in view of the fact that most magnetic ultrathin films investigated experimentally consist of metals and alloys.

In the present paper, I extend the result of Mermin and Wagner to Heisenberg and $X Y$ systems with a long-range interaction, i.e., which do not satisfy the condition (1), with particular emphasis on systems with oscillatory interactions. Results for systems with monotonically decaying interactions are also presented. More specifically, I consider one- and two-dimensional systems described by the following Hamiltonian:

$$
\begin{aligned}
H= & -\sum_{\mathbf{R}, \mathbf{R}^{\prime}}\left[J_{\mathbf{R}-\mathbf{R}^{\prime}}^{\prime} S_{\mathbf{R}^{x}}^{x} S_{\mathbf{R}^{\prime}}^{x}+J_{\mathbf{R}-\mathbf{R}^{\prime}}\left(S_{\mathbf{R}}^{y} S_{\mathbf{R}^{\prime}}^{y}+S_{\mathbf{R}}^{z} S_{\mathbf{R}^{\prime}}^{z}\right)\right] \\
& +K \sum_{\mathbf{R}}\left(S_{\mathbf{R}}^{x}\right)^{2}-B \sum_{\mathbf{R}} S_{\mathbf{R}}^{z}
\end{aligned}
$$

We also define the Fourier transform of the spin operator $\quad \mathbf{S}_{\mathbf{R}}, \quad \mathbf{S}(\mathbf{k}) \equiv \sum_{\mathbf{R}} \mathbf{S}_{\mathbf{R}} \mathrm{e}^{-\mathrm{ik} \cdot \mathbf{R}}, \quad$ as well as $E(\mathbf{k}) \equiv \sum_{\mathbf{R}} J_{\mathbf{R}}\left(1-\mathrm{e}^{\mathrm{i} \mathbf{k} \cdot \mathbf{R}}\right)=E(-\mathbf{k}), \quad$ and $\quad \tilde{E}(\mathbf{k}) \equiv$ $\sum_{\mathbf{R}}\left|J_{\mathbf{R}}\right|\left(1-\mathrm{e}^{\mathrm{i} \mathbf{k} \cdot \mathbf{R}}\right)=\tilde{E}(-\mathbf{k})$. For $K=0$ and $J_{\mathbf{R}}^{\prime}=$ $J_{\mathbf{R}}$, the above Hamiltonian corresponds to the isotropic Heisenberg model. In the general case, it corresponds to a system with uniaxial anisotropy of axis $x$, with a single-spin anisotropy (for $S \geq 1$ ) and/or a two-spin exchange anisotropy. Depending on the value of the anisotropy parameters $K$ and $J_{\mathbf{R}}^{\prime}-J_{\mathbf{R}}$, one therefore has either an isotropic system, an $X Y$-like system with $y z$ easy plane, or an Ising-like model with $x$ easy axis (in the latter case, one merely shows that the spontaneous magnetization has to be along the $x$ axis). For such systems (with arbitrary values of parameters $J_{\mathbf{R}}^{\prime}$ and $K$ ), I prove the following results:

Theorem 1. - A $D$-dimensional $(D=1$ or 2$)$ Heisenberg or $X Y$ system for which one can find some numbers $k_{0}>0$ [with $k_{0}$ belonging to the first Brillouin zone (BZ)], $\alpha>0$, $\beta \leq 1$, such that, for $|\mathbf{k}|<k_{0}$ and $\left|\mathbf{k}^{\prime}\right|<k_{0}$,

$$
\begin{aligned}
&|E(\mathbf{k})| \leq \alpha|\mathbf{k}|^{D}\left|\ln \left(|\mathbf{k}|^{-1}\right)\right|^{\beta} \\
& \mid E\left(\mathbf{k}^{\prime}-\mathbf{k}\right)+E\left(\mathbf{k}^{\prime}+\mathbf{k}\right)-2 E\left(\mathbf{k}^{\prime}\right) \mid \\
& \leq \alpha|\mathbf{k}|^{D}\left|\ln \left(|\mathbf{k}|^{-1}\right)\right|^{\beta} \\
& \frac{1}{A_{D}} \int d^{D} \mathbf{k}^{\prime \prime} \mid E\left(\mathbf{k}^{\prime \prime}-\mathbf{k}\right)+ E\left(\mathbf{k}^{\prime \prime}+\mathbf{k}\right)-2 E\left(\mathbf{k}^{\prime \prime}\right) \mid \\
& \leq \alpha|\mathbf{k}|^{D}\left|\ln \left(|\mathbf{k}|^{-1}\right)\right|^{\beta}
\end{aligned}
$$

cannot be ferromagnetic [6].

Corollary 1. - A $D$-dimensional $(D=1$ or 2$)$ Heisenberg or $X Y$ system with long-range oscillatory interactions of the form

$$
J_{\mathbf{R}} \propto \frac{\cos \left(q_{0} R+\phi\right)}{R^{\alpha}},
$$


with $q_{0} \neq 0$ belonging to the first BZ and $\alpha \geq 1(D=1)$, or $\alpha>3 / 2[D=2, \sin (\phi+\pi / 4)=0]$, or $\alpha>5 / 2[D=$ $2, \sin (\phi+\pi / 4) \neq 0]$ cannot be ferromagnetic.

Theorem 2.-A $D$-dimensional $(D=1$ or 2$)$ Heisenberg or $X Y$ system satisfying

$$
\frac{1}{A_{D}} \int d^{D} \mathbf{k} \frac{1}{\tilde{E}(\mathbf{k})}=+\infty,
$$

where $A_{D}$ is the measure of the first BZ, cannot be ferroor antiferromagnetic.

Corollary 2.-A $D$-dimensional ( $D=1$ or 2) Heisenberg or $X Y$ system with interactions monotonically decaying as $\left|J_{\mathbf{R}}\right| \propto R^{-\alpha}$ with $\alpha \geq 2 D$ cannot be ferro- or antiferromagnetic.

Proof of Theorem 1.-As for the Mermin-Wagner theorem, the proof of Theorem 1 relies on using the Bogoliubov inequality to prove that the $z$ component of the spontaneous magnetization $\lim _{B \rightarrow 0}\left|\left\langle S^{z}\right\rangle\right|$ vanishes for any finite temperature. The proof of Theorem 1 is, however, significantly more delicate than the Mermin-Wagner one.

Our proof proceeds by reductio ad absurdum. Let us assume that the system is ferromagnetic. This implies that one can find a temperature $T_{0}>0$ and a quantity $m_{0}>0$ such that, for any temperature $T \leq T_{0}$, one has $\left|\left\langle S^{z}\right\rangle\right| \geq m_{0}$.

The Bogoliubov inequality [1] states that $\left\langle\left\{A, A^{\dagger}\right\}\right\rangle\left\langle\left[[C, H], C^{\dagger}\right]\right\rangle \geq 2 k_{B} T|\langle[C, A]\rangle|^{2}$, where $A$ and $C$ are arbitrary operators, $[A, B]$ is the commutator of $A$ and $B,\{A, B\}$ is the anticommutator of $A$ and $B$, and $\langle A\rangle$ is the thermodynamic average of $A$. The two factors on the left-hand side of the inequality are $\geq 0$. We use Bogoliubov's inequality for $A \equiv S^{y}(-\mathbf{k})$ and $C \equiv S^{x}(\mathbf{k})$. Straightforward algebra together with the inequality $\left\langle\left(S_{\mathbf{R}}^{y}\right)^{2}\right\rangle \leq S^{2}$ then yield the following inequality:

$$
S^{2} \geq \frac{1}{A_{D}} \int d^{D} \mathbf{k} \frac{k_{B} T\left\langle S^{z}\right\rangle^{2}}{B\left\langle S^{z}\right\rangle+2 \sum_{\mathbf{R}} J_{\mathbf{R}}[1-\cos (\mathbf{k} \cdot \mathbf{R})]\left\langle S_{0}^{y} S_{\mathbf{R}}^{y}+S_{0}^{z} S_{\mathbf{R}}^{z}\right\rangle} .
$$

Note that the above result is independent of $J_{\mathbf{R}}^{\prime}$ and $K$, and depends only on $J_{\mathbf{R}}$. Thus far, our proof follows exactly the one of Mermin and Wagner. From this point, Mermin and Wagner proceed by stating that, if the condition (1) is satisfied, the (positive) denominator $\Delta(\mathbf{k})$ of the above equation can be majorated near $\mathbf{k}=0$ by an expression of the form $|B| S+\alpha k^{2}$ (with $\alpha>0$ ), from which they then easily show that the spontaneous magnetization vanishes.

In order to obtain a stronger theorem, i.e., to rule out ferromagnetism for a broader class of systems, one therefore needs to find a tighter majoration of $\Delta(\mathbf{k})$ than used by Mermin and Wagner. One can check easily that a majoration of the form $\Delta(\mathbf{k}) \leq|B| S+\gamma|\mathbf{k}|^{D}\left|\ln \left(|\mathbf{k}|^{-1}\right)\right|^{\beta}$ near $\mathbf{k}=0$, with $\gamma>0$ and $\beta \leq 1$, would be sufficient for our purpose.

To this aim, we rewrite the denominator $\Delta(\mathbf{k})$ of Eq. (6) as

$$
\begin{aligned}
& \Delta(\mathbf{k})=B\left\langle S^{z}\right\rangle+2\left\langle S^{z}\right\rangle^{2} E(\mathbf{k}) \\
&+\frac{1}{A_{D}} \int d^{D} \mathbf{k}^{\prime} {\left[E\left(\mathbf{k}^{\prime}-\mathbf{k}\right)\right.} \\
&\left.+E\left(\mathbf{k}^{\prime}+\mathbf{k}\right)-2 E\left(\mathbf{k}^{\prime}\right)\right] F\left(\mathbf{k}^{\prime}\right),
\end{aligned}
$$

with

$$
\begin{aligned}
F(\mathbf{k}) & \equiv \sum_{\mathbf{R}} \mathrm{e}^{-\mathrm{i} \mathbf{k} \cdot \mathbf{R}}\left\langle S_{0}^{y} S_{\mathbf{R}}^{y}+\delta S_{0}^{z} \delta S_{\mathbf{R}}^{z}\right\rangle \\
& =\frac{1}{N}\left\langle\left|S^{y}(\mathbf{k})\right|^{2}+\left|\delta S^{z}(\mathbf{k})\right|^{2}\right\rangle \\
& =k_{B} T\left[\chi^{y y}(\mathbf{k})+\chi^{z z}(\mathbf{k})\right],
\end{aligned}
$$

where we have introduced the longitudinal fluctuation $\delta S_{\mathbf{R}}^{z} \equiv S_{\mathbf{R}}^{z}-\left\langle S^{z}\right\rangle$ and the transverse and longitudinal $\mathbf{k}$-dependent susceptibilities $\chi^{y y}(\mathbf{k})$ and $\chi^{z z}(\mathbf{k})$, respectively. Since we are assuming that the system has a long-range ferromagnetic order, one can argue that $F(\mathbf{k})$ has to be finite for $\mathbf{k} \neq 0$; otherwise the ferromagnetic order would be unstable against fluctuations of the nonzero wave vector. On the other hand, $F(\mathbf{k})$ certainly diverges for $\mathbf{k}=0$ due to the divergence of the static transverse susceptibility of systems having a continuous rotational invariance. Nevertheless, one has

$$
\frac{1}{A_{D}} \int d^{D} \mathbf{k} F(\mathbf{k})=\left\langle\left(S^{y}\right)^{2}+\left(S^{z}\right)^{2}\right\rangle \leq S(S+1) .
$$

In view of the above remarks concerning $F(\mathbf{k})$, there exists a number $F_{0}>0$ such that $0 \leq F(\mathbf{k}) \leq F_{0}$ for $|\mathbf{k}|>k_{0}$. Making use of conditions (3b) and (3c), one can therefore write, for $|\mathbf{k}|<k_{0}$,

$$
\begin{aligned}
A(\mathbf{k}) \equiv & \frac{1}{A_{D}} \int d^{D} \mathbf{k}^{\prime}\left|E\left(\mathbf{k}^{\prime}-\mathbf{k}\right)+E\left(\mathbf{k}^{\prime}+\mathbf{k}\right)-2 E\left(\mathbf{k}^{\prime}\right)\right| F\left(\mathbf{k}^{\prime}\right) \\
& \leq \frac{1}{A_{D}} \int_{\left|\mathbf{k}^{\prime}\right|<k_{0}} d^{D} \mathbf{k}^{\prime} \alpha|\mathbf{k}|^{D}\left|\ln \left(|\mathbf{k}|^{-1}\right)\right|^{\beta} F\left(\mathbf{k}^{\prime}\right)+\frac{1}{A_{D}} \int_{\left|\mathbf{k}^{\prime}\right|>k_{0}} d^{D} \mathbf{k}^{\prime}\left|E\left(\mathbf{k}^{\prime}-\mathbf{k}\right)+E\left(\mathbf{k}^{\prime}+\mathbf{k}\right)-2 E\left(\mathbf{k}^{\prime}\right)\right| F_{0} \\
& \leq \frac{1}{A_{D}} \int d^{D} \mathbf{k}^{\prime} \alpha|\mathbf{k}|^{D}\left|\ln \left(|\mathbf{k}|^{-1}\right)\right|^{\beta} F\left(\mathbf{k}^{\prime}\right)+\frac{1}{A_{D}} \int d^{D} \mathbf{k}^{\prime}\left|E\left(\mathbf{k}^{\prime}-\mathbf{k}\right)+E\left(\mathbf{k}^{\prime}+\mathbf{k}\right)-2 E\left(\mathbf{k}^{\prime}\right)\right| F_{0} \\
& \leq\left[S(S+1)+F_{0}\right] \alpha|\mathbf{k}|^{D}\left|\ln \left(|\mathbf{k}|^{-1}\right)\right|^{\beta}
\end{aligned}
$$


Combining this result with condition (3a), we obtain

$$
0 \leq \Delta(\mathbf{k}) \leq|B| S+\gamma|\mathbf{k}|^{D}\left|\ln \left(|\mathbf{k}|^{-1}\right)\right|^{\beta},
$$

for $|\mathbf{k}|<k_{0}$, with $\gamma \equiv S^{2}+S(S+1)+F_{0}>0$. Combining the above result with inequality (6), one obtains

$$
S^{2} \geq \frac{1}{A_{D}} \int_{\left|\mathbf{k}^{\prime}\right|<k_{0}} d^{D} \mathbf{k}^{\prime} \frac{k_{B} T\left\langle S^{z}\right\rangle^{2}}{|B| S+\gamma|\mathbf{k}|^{D}\left|\ln \left(|\mathbf{k}|^{-1}\right)\right|^{\beta}},
$$

from which one shows immediately that $\lim _{B \rightarrow 0}\left|\left\langle S^{z}\right\rangle\right|=0$ for $T>0$, which is in contradiction with our hypothesis that the system is ferromagnetic. This completes the proof of Theorem 1 by reductio ad absurdum.

Proof of Corollary 1.-Consider interaction of the form of Eq. (4). To investigate the behavior of $E(\mathbf{k})$ in the vicinity of $\mathbf{k}=0$, we can substitute the discrete sum over $\mathbf{R}$ by an integral. One can show easily that, for any value of $\alpha, E(\mathbf{k})$ is analytical for all wave vectors except for $|\mathbf{k}|=q_{0}$. Therefore, for $\mathbf{k} \rightarrow 0$,

$$
E(\mathbf{k}) \propto|\mathbf{k}|^{2} \quad(\forall \alpha),
$$

and, for $\mathbf{k} \rightarrow 0$ and $|\mathbf{k}|<|| \mathbf{k}^{\prime}\left|-q_{0}\right|$,

$$
\left|E\left(\mathbf{k}^{\prime}-\mathbf{k}\right)+E\left(\mathbf{k}^{\prime}+\mathbf{k}\right)-2 E\left(\mathbf{k}^{\prime}\right)\right| \propto|\mathbf{k}|^{2} \quad(\forall \alpha) .
$$

The integral over $\mathbf{k}^{\prime \prime}$ in (3c) is dominated by the nonanalyticity of $E(\mathbf{k})$ in the vicinity of $\left|\mathbf{k}^{\prime \prime}\right|=q_{0}$. Let us therefore study $\varepsilon(\kappa)$, the singular part of $E(\mathbf{k})$ for $\kappa \equiv|\mathbf{k}|-q_{0} \rightarrow$ 0 . We get

$$
\varepsilon(\kappa) \propto \begin{cases}{[A+B \operatorname{sgn}(\kappa)]|\kappa|^{\alpha-1}} & (\alpha<1) \\ A \ln |\kappa|^{-1}+B \operatorname{sgn}(\kappa) & (\alpha=1) \\ B \operatorname{sgn}(\kappa)|\kappa|^{\alpha-1} & (1<\alpha<2) \\ B \operatorname{sgn}(\kappa)|\kappa| \ln |\kappa|^{-1} & (\alpha=2) \\ B \operatorname{sgn}(\kappa)|\kappa| & (\alpha>2),\end{cases}
$$

for $D=1$, with $A \propto \cos \phi$ and $B \propto \sin \phi$, and

$$
\varepsilon(\kappa) \propto \begin{cases}{[A+B \operatorname{sgn}(\kappa)]|\kappa|^{\alpha-3 / 2}} & (\alpha<3 / 2) \\ A \ln |\kappa|^{-1}+B \operatorname{sgn}(\kappa) & (\alpha=3 / 2) \\ B \operatorname{sgn}(\kappa)|\kappa|^{\alpha-3 / 2} & (3 / 2<\alpha<5 / 2) \\ B \operatorname{sgn}(\kappa)|\kappa| \ln |\kappa|^{-1} & (\alpha=5 / 2) \\ B \operatorname{sgn}(\kappa)|\kappa| & (\alpha>5 / 2),\end{cases}
$$

for $D=2$, with $A \propto \cos (\phi+\pi / 4)$ and $B \propto$ $\sin (\phi+\pi / 4)$. Let $C(\mathbf{k})$ be the left-hand side expression in Eq. (3c). We then get, for $\mathbf{k} \rightarrow 0$,

$$
C(k) \propto \begin{cases}+\infty & (\alpha \leq 0) \\ |k|^{\alpha} & (0<\alpha<1) \\ |k| \ln |k|^{-1} & (\alpha=1, \cos \phi \neq 0) \\ |k| & (\alpha=1, \cos \phi=0) \\ |k|^{2} & (\alpha>1, \sin \phi=0) \\ |k| & (1<\alpha<2, \sin \phi \neq 0) \\ |k|^{2} \ln |k|^{-1} & (\alpha=2, \sin \phi \neq 0) \\ |k|^{2} & (\alpha>2, \sin \phi \neq 0)\end{cases}
$$

for $D=1$, and

$$
C(\mathbf{k}) \propto \begin{cases}+\infty & (\alpha \leq 1 / 2) \\ |\mathbf{k}|^{\alpha-1 / 2} & (1 / 2<\alpha<3 / 2) \\ |\mathbf{k}| \ln |\mathbf{k}|^{-1} & (\alpha=3 / 2, \cos (\phi+\pi / 4) \neq 0) \\ |\mathbf{k}| & (\alpha=3 / 2, \cos (\phi+\pi / 4)=0) \\ |\mathbf{k}|^{2} & (\alpha>3 / 2, \sin (\phi+\pi / 4)=0) \\ |\mathbf{k}|^{\alpha-1 / 2} & (3 / 2<\alpha<5 / 2, \sin (\phi+\pi / 4) \neq 0) \\ |\mathbf{k}| \ln |\mathbf{k}|^{-1} & (\alpha=5 / 2, \sin (\phi+\pi / 4) \neq 0) \\ |\mathbf{k}|^{2} & (\alpha>5 / 2, \sin (\phi+\pi / 4) \neq 0)\end{cases}
$$

for $D=2$. From Eqs. (13) and (14), it follows that conditions (3a) and (3b) are satisfied for all values of $\alpha$. From Eqs. (17) and (18), one gets that condition (3c) is fulfilled for $\alpha \geq 1(D=1)$, and for $\alpha>3 / 2[D=2, \sin (\phi+\pi / 4)=0]$ or $\alpha>$ $5 / 2[D=2, \sin (\phi+\pi / 4) \neq 0]$, which completes the proof of Corollary 1.

Proof of Theorem 2. - Theorem 2 is an immediate generalization of the Mermin-Wagner theorem. For the sake of simplicity, we detail the proof only for the case of ferromagnetism; the extension to the case of antiferromagnetism is immediate by introducing a staggered field and staggered magnetization as done by Mermin and Wagner.

The proof follows the one of Theorem 1 until Eq. (6). Then, by using $\left|\left\langle S_{0}^{y} S_{\mathbf{R}}^{y}+S_{0}^{z} S_{\mathbf{R}}^{z}\right\rangle\right| \leq S(S+1)$,

$$
\Delta(\mathbf{k}) \leq|B| S+2 S(S+1) \tilde{E}(\mathbf{k}) .
$$

From condition (5) it follows immediately that $\lim _{B \rightarrow 0}\left|\left\langle S^{z}\right\rangle\right|=0$ for $T>0$, which is in contradiction with our hypothesis that the system is ferromagnetic. This completes the proof of Theorem 2 by reductio ad absurdum.

Proof of Corollary 2. - Let us consider the behavior of $\tilde{E}(\mathbf{k})$ in the vicinity of $\mathbf{k}=0$ for $\left|J_{\mathbf{R}}\right| \propto R^{-\alpha}$. In this regime, the discrete sum over $\mathbf{R}$ can be replaced by an integral, and one shows easily that, for $|\mathbf{k}| \rightarrow 0$,

$$
\tilde{E}(\mathbf{k}) \propto \begin{cases}|k|^{\alpha-D} & (D<\alpha<D+2) \\ |k|^{2} \ln \left(|k|^{-1}\right) & (\alpha=D+2) \\ |k|^{2} & (\alpha>D+2) .\end{cases}
$$

It then follows immediately that condition (5) is satisfied if $\alpha \geq 2 D$, which completes the proof of Corollary 2 .

As for the Mermin-Wagner theorem, the above theorems and corollaries can be extended also to 
one-dimensional systems of arbitrary finite cross section and to two-dimensional systems of arbitrary finite thickness.

The importance of Theorem 1 and Corollary 1 is that the class of systems for which ferromagnetism is rigorously ruled out is significantly increased. In particular, we obtain interesting results for systems with RKKY interactions $(\alpha=D)$. For one-dimensional RKKY systems, ferromagnetism is rigorously ruled out. For twodimensional RKKY systems, the results are less satisfactory, since ferromagnetism can be strictly ruled out only for the particular case with $\sin (\phi+\pi / 4)=0$. For the general case $[\sin (\phi+\pi / 4) \neq 0]$, it does not seem possible to rule out ferromagnetism for $\alpha=D=2$ without analyzing in detail the behavior of $F(\mathbf{k})$ near $|\mathbf{k}|=q_{0}$. If one could prove that $F(\mathbf{k})$ has no singularity more singular than a square-root singularity at $|\mathbf{k}|=q_{0}$, then the absence of ferromagnetism would follow from Eq. (18). This situation is somehow puzzling. Indeed, within the (nonrigorous) linearized spin-wave theory, as well as the random phase approximation Green's function method, having $E(\mathbf{k}) \propto k^{2}$ is sufficient to rule out ferromagnetism in two dimensions, which implies that these theories rule ferromagnetism in systems with oscillatory interactions, for any value of $\alpha$. In view of these considerations, I therefore propose the following result:

Conjecture 1.-A $D$-dimensional ( $D=1$ or 2$)$ Heisenberg or $X Y$ system, for which there exists some finite $\mathbf{k}$ region with $E(\mathbf{k})<0$, or satisfying

$$
\frac{1}{A_{D}} \int d^{D} \mathbf{k} \frac{1}{E(\mathbf{k})}=+\infty
$$

cannot be ferromagnetic.

From Eq. (13), it then follows immediately:

Corollary 3. - If Conjecture 1 is true, then a $D$ dimensional ( $D=1$ or 2 ) Heisenberg or $X Y$ system with long-range oscillatory interactions of the form (4) cannot be ferromagnetic, for any value of $\alpha$.

Let us now discuss the results of Theorem 2 and Corollary 2. They essentially constitute an extension to $X Y$ systems of a result obtained earlier by Joyce [7]. For the one-dimensional (classical) Heisenberg and $X Y$ systems with positive (ferromagnetic) long-range interaction, Fröhlich et al. proved the existence of ferromagnetism if $1<\alpha<2$ [8]. This is to be contrasted with onedimensional Ising with long-range interaction, for which ferromagnetism is ruled out if $\alpha>2$ [9], whereas ferromagnetism exists for $1<\alpha \leq 2$ [10]. The Heisenberg and $X Y$ systems therefore differ only from the Ising system on the borderline $\alpha=2$ : there ferromagnetism is excluded for the Heisenberg and $X Y$ cases, but exists for the Ising case, illustrating the stronger tendency towards ordering displayed by the Ising model.

I wish to express my thanks to V. Dugaev and G. Bouzerar for helpful discussions, as well as to A.C.D. van Enter and E. Luijten for critical comments on an early version of this Letter.

*Electronic address: bruno@mpi-halle.de ${ }^{\dagger}$ http://www.mpi-halle.de

[1] N. N. Bogoliubov, Phys. Abhandl. Sowjetunion 6, 1 (1962).

[2] N. D. Mermin and H. Wagner, Phys. Rev. Lett. 17, 1133 (1966).

[3] For the sake of concision throughout this paper, a system will be termed "ferromagnetic" (respectively, "antiferromagnetic") (or not) without further precision if it possesses (or not) ferromagnetic (respectively, antiferromagnetic) long-range order below some nonzero Curie temperature, without prejudice of the magnetic order exhibited in the ground state.

[4] D. Ruelle, Statistical Mechanics: Rigorous Results (Benjamin, New York, 1969); R. B. Griffiths, in Phase Transitions and Critical Phenomena, edited by C. Domb and M. S. Green (Academic, New York, 1972), Vol. 1, p. 7; B. Simon, The Statistical Mechanics of Lattice Gases (Princeton University Press, Princeton, NJ, 1993), Vol. 1.

[5] M. A. Ruderman and C. Kittel, Phys. Rev. 96, 99 (1954); T. Kasuya, Prog. Theor. Phys. 16, 45 (1956); K. Yosida, Phys. Rev. 106, 893 (1957).

[6] The continuous integration over wave vectors indicates that we are taking the thermodynamic limit. Throughout the paper, all integrations over wave vectors are strictly restricted to the first BZ, unless explicitly further restricted.

[7] G. S. Joyce, J. Phys. C, Solid State Phys. 2, 1531 (1969).

[8] J. Fröhlich, R. Israel, E. H. Lieb, and B. Simon, Commun. Math. Phys. 62, 1 (1978).

[9] D. Ruelle, Commun. Math. Phys. 9, 267 (1968).

[10] F. J. Dyson, Commun. Math. Phys. 12, 91 (1969); J. Fröhlich and T. Spencer, Commun. Math. Phys. 84, 87 (1982). 